\title{
Triplet Distribution Functions and their Superposition Approximation from an MD-simulation of molten $\mathrm{KCl}$
}

\author{
L. Schäfer and A. Klemm \\ Max-Planck-Institut für Chemie (Otto-Hahn-Institut), Mainz
}

Z. Naturforsch. 36a, 584-587 (1981); received April 20, 1981

\begin{abstract}
Isosceles triplet distribution functions of molten $\mathrm{KCl}$ are obtained from an $\mathrm{MD}$-simulation and compared with those approximated by multiplication of the corresponding three pair distribution functions. For small branch lengths $r$ the deviations are considerable, e.g. for $r=3 \AA$ the frequency of linear triplets +-+ or -+- relative to the frequency of all triplets with that branch length is underestimated by a factor of about 0.6 .
\end{abstract}

With the present art of $x$-ray and neutron diffraction analysis the triplet distribution functions (TDF's) of liquids are inaccessible. These functions can, however, be obtained from computer simulations and have been evaluated for monoatomic liquids [1]-[7]. For diatomic liquids there exists only the paper on molten $\mathrm{KCl}$ [8], in which the relative frequencies of angles with branches corresponding in length to the first maxima of the pair distribution functions (PDF's) are given. The present paper is a continuation of [8]. The lengths of the two branches and the ions at the ends of these are taken to be equal, and the lengths are varied in steps. The TDF's in the superposition approximation are also calculated for comparison.

\section{General}

The TDF's of liquids are defined in this paper as the occupation numbers of triangular configurations of three atoms, specified by their kinds, as functions of three independent variables chosen to describe the triangles, divided by these numbers in case of a distribution of noninteracting point particles.

In general, if two lengths $(r$ and $R$ ) and an angle $(\vartheta)$ are chosen as the variables to describe the triangles, and if the atoms of the triplet are of different kinds (A, B and C), the corresponding TDF can be written in the form $g_{\mathrm{ABC}}(r, R, \vartheta)$, where the first and second lengths are the distances of the first and second atoms from the third atom, re-

Reprint requests to Prof. Dr. A. Klemm, Saarstraße 23, D-65 Mainz. spectively, and $\vartheta$ is the angle at the third atom. If the liquid consists of two kinds of atoms (A and B), then the four TDF's $g_{\mathrm{AAA}}(r, R, \vartheta), g_{\mathrm{BBB}}(r, R, \vartheta)$, $g_{\mathrm{BBA}}(r, R, \vartheta)$ and $g_{\mathrm{AAB}}(r, R, \vartheta)$ give all possible information about the triplet correlations in the liquid.

Within the accuracy of our simulation, due to the similarity of the pair potentials $\mathrm{K}^{+}-\mathrm{K}^{+}$and $\mathrm{Cl}^{-}-$ $\mathrm{Cl}^{-}$the above TDF's are essentially invariant against an exchange of $\mathrm{A}$ and $\mathrm{B}$, i.e.

$$
\begin{aligned}
& g_{\mathrm{AAA}}(r, R, \vartheta) \approx g_{\mathrm{BBB}}(r, R, \vartheta) \equiv g_{11}(r, R, \vartheta), \\
& g_{\mathrm{BBA}}(r, R, \vartheta) \approx g_{\mathrm{AAB}}(r, R, \vartheta) \equiv g_{\mathrm{uu}}(r, R, \vartheta) .
\end{aligned}
$$

For convenience we write $g_{11}$ for the first and $g_{\text {uu }}$ for the second TDF, where the subscripts 1 and $u$ indicate if the ions at the ends of the two branches are like or unlike the ion at the vertex. Similarly we write $g_{1}(r)$ and $g_{\mathrm{u}}(r)$ for the PDF's because in our case $g_{\mathrm{AA}}(r) \approx g_{\mathrm{BB}}(r)$.

As the physical interest in the structure of ionic liquids is centered on the spherical surroundings of the ions, we have restricted our calculations to the projections $g_{11}(r, r, \vartheta)$ and $g_{\mathrm{uu}}(r, r, \vartheta)$ of the TDF's $g_{11}(r, R, \vartheta)$ and $g_{\mathrm{uu}}(r, R, \vartheta)$, i.e. we have only considered isosceles triangles.

The TDF's obtained from our MD simulations shall be compared with approximate TDF's constructed by multiplication of the corresponding PDF's obtained from the same simulation. The TDF's in this approximation, the so called superposition approximation (SA), shall be marked with a prime. On has in our case

$$
\begin{aligned}
& g_{\mathrm{ss}}^{\prime}(r, r, \vartheta)=g_{\mathrm{s}}(r) g_{\mathrm{s}}(r) g_{\mathrm{l}}(2 r \sin (\vartheta / 2)), \\
& \mathrm{s}=\mathrm{u} \text { or } 1 .
\end{aligned}
$$

0340-4811 / $81 / 0600-0584 \$ 01.00 / 0$. - Please order a reprint rather than making your own copy. 
The justification and limitation of the SA are as follows:

The probability that three pairs of atoms with large interpair distances have the intrapair distances $r, s$ and $t$ is proportional to the product of the corresponding values of the corresponding three PDF's. The value of this product approaches unity with increasing intrapair distances, as does the value of the TDF. Therefore the approximated TDF has the right normalisation to be compared with the true TDF. In the SA the fact is ignored, however, that the three pairs are interlocked to form a triplet. A correction to the $\mathrm{SA}$ for monotomic liquids is given in Ref. [9].

The ratios of the true TDF's to the TDF's in the uncorrected SA are of interest and are called triplet correlation functions. Raveché $[4,5]$ uses the symbol $g_{3}$ for this ratio.

\section{Distribution Functions from the MD-Simulation}

In order to avoid having periodically repeated particles in the spheres around the reference particles used for the determination of the PDF's, $r$ was restricted to $r<10 \AA$, which is half the sidelength of the periodic cube of our simulation. This involves that the complete angle dependence of the functions $g_{\mathrm{ss}}^{\prime}$ could only be calculated for $r<5 \AA$, and consequently the calculation of $g_{\mathrm{ss}}$ has been restricted to this range too.

For the evaluation of the functions $g_{\mathrm{ss}}, n_{\mathrm{T}}=4800$ configurations of the MD simulation, $0.0025 \mathrm{ps}$ apart in time, were used. For every one of the $n_{\mathrm{T}}$ configurations, each of the $2 n_{\mathrm{J}}=216$ ions in the periodic cube was taken as the reference ion, and the angles $\vartheta$ of all the triangles formed by the $n_{\mathrm{T}} 2 n_{\mathrm{J}}$ reference ions and all possible ion pairings of the respective kind of ions in shells of breadth $\Delta r=$ $0.1 \AA$ around the reference ions were stored with an increment $\Delta \vartheta=\pi / 180$. Triplets of like ions forming equilateral triangles are thus counted as three triplets forming isoscles triangles.

Let $\Delta n_{\mathrm{ss}}$ be the number of triplets found in this way for a range $r \pm \frac{1}{2} \Delta r, \vartheta \pm \frac{1}{2} \Delta \vartheta$. Then $g_{\mathrm{ss}}$ is given by

$$
\begin{aligned}
& g_{\mathrm{ss}}(r, r, \vartheta)=\Delta n_{\mathrm{ss}}(r, r, \vartheta) / \Delta n_{0 \mathrm{ss}}(r, r, \vartheta) \\
& \mathrm{s}=\mathrm{u} \text { or } 1,
\end{aligned}
$$

where $\Delta n_{0 \mathrm{ss}}$ is the number of triplets which would have been found if the distribution of the particles in the periodic cube would have been at all times a distribution of $2 n_{\mathrm{J}}$ noninteracting point particles. Indeed, by this definition $g_{\text {ss }}$ equals unity for the zero interaction distribution.

$\Delta n_{0 \text { ss }}$ is found by the following reasoning: Let $V$ be the volume of the periodic cube containing $n_{\mathrm{J}}$ cations and $n_{\mathrm{J}}$ anions, $\Delta V$ the volume of a shell of the range $r \pm \frac{1}{2} \Delta r$, and $\Delta^{2} V$ the volume of a zone of the range $r \pm \frac{1}{2} \Delta r, \vartheta \pm \frac{1}{2} \Delta \vartheta$. Then for zero interaction $n_{\mathrm{J}} \Delta V / V$ is the average number of unlike ions in a shell around an ion and $\left(n_{J}-1\right) \Delta V / V$ the corresponding number of like ions, the reference ion for placing a shell never being in that shell. Further, $\left(n_{J}-1\right) \Delta^{2} V / V$ is the average number of unlike ions in a zone of a shell, the reference ion for placing a zone never being in that zone, and $\left(n_{J}-2\right) \Delta^{2} V / V$ is the corresponding number of like ions, the reference ion for placing a shell and the reference ion for placing a zone never being in that zone.

\section{One arrives at}

$$
\begin{aligned}
& \Delta n_{0 \mathrm{ss}}(r, r, \vartheta) \\
& =\frac{1}{2} n_{\mathrm{T}} 2 n_{\mathrm{J}} v_{\mathrm{s}}\left(v_{\mathrm{s}}-1\right) \Delta V \Delta^{2} V / V^{2}, \\
& \mathrm{~s}=\mathrm{u} \text { or } 1,
\end{aligned}
$$

with $v_{\mathrm{u}}=n_{\mathrm{J}}$ and $v_{\mathrm{l}}=n_{\mathrm{J}}-1$. The factor $\frac{1}{2}$ in (4) is necessary to avoid counting the pairs in a shell twice. In differential form

$$
\begin{aligned}
\mathrm{d} V & =4 \pi r^{2} \mathrm{~d} r, \\
\mathrm{~d}^{2} V & =2 \pi r^{2} \mathrm{~d} r \sin \vartheta \mathrm{d} \vartheta .
\end{aligned}
$$

For a given sidelength $r$, the relative number of triplets forming triangles with angles $\vartheta$ within $\mathrm{d} \vartheta$ is

$$
\begin{aligned}
& {\left[\frac{\partial}{\partial \vartheta} n_{\mathrm{ss}}(r, r, \vartheta)\right] \mathrm{d} \vartheta / \int_{0}^{\pi}\left[\frac{\partial}{\partial \vartheta} n_{\mathrm{ss}}(r, r, \vartheta)\right] \mathrm{d} \vartheta} \\
& \equiv H_{\mathrm{SS}}(r, r, \vartheta) \mathrm{d} \vartheta
\end{aligned}
$$

where $H_{\mathrm{ss}}$ follows from (3)-(6) to be

$$
H_{\mathrm{ss}}(r, r, \vartheta)=g_{\mathrm{ss}}(r, r, \vartheta) \sin \vartheta /\left[2 G_{\mathrm{ss}}(r, r)\right]
$$

with

$$
G_{\mathrm{ss}}(r, r)=\frac{1}{2} \int_{0}^{\pi} g_{\mathrm{ss}}(r, r, \vartheta) \sin \vartheta \mathrm{d} \vartheta .
$$

Since $g_{\mathrm{ss}}$ equals unity for the case of zero particle interaction and

$$
\int_{0}^{\pi} \sin \vartheta \mathrm{d} \vartheta
$$

equals two, $G_{\mathrm{ss}}(r, r)$ is the number of triplets forming isosceles triangles with the side length $r$ 


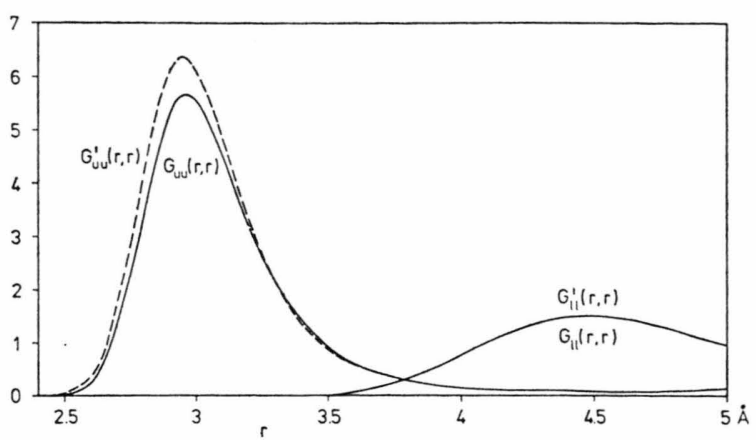

Fig. 1. TDF's, integrated over the angle according to (9). The curves approach unity with increasing $r$ and become horizontal lines, 1 vs. $r$, for noninteracting particles. Broken lines correspond to the Superposition Approximation here and in Figs. 2 and 3.

divided by this number in case of zero particleinteraction. In Fig. 1 the functions $G_{\mathrm{uu}}, G_{\mathrm{uu}}^{\prime}, G_{11}$, and $G_{11}^{\prime}$ are plotted vs. $r$.

A plot of $H_{\mathrm{ss}}$ vs. $\vartheta$ would have the disadvantage that zero particle-interaction would be represented by a curve $\left(\frac{1}{2} \sin \vartheta\right.$ vs. $\vartheta$ ), and that $H_{\text {ss }}$ vanishes for $\vartheta=\pi$. Now, the r. h. s. of (7) can be written in the form

$$
H_{\mathrm{ss}}(r, r, \vartheta) \mathrm{d} \vartheta=-h_{\mathrm{Ss}}(r, r, \vartheta) \mathrm{d} \cos \vartheta
$$

with

$$
h_{\mathrm{ss}}(r, r, \vartheta)=H_{\mathrm{ss}}(r, r, \vartheta) / \sin \vartheta .
$$

In a plot of $h_{\mathrm{ss}}$ vs. $\cos \vartheta$ both disadvantages disappear: Zero particle-interaction is represented by a straight line $\left(\frac{1}{2}\right.$ vs. $\left.\cos \vartheta\right)$ and $h_{\mathrm{ss}}$ does not disappear at $\vartheta=\pi$.

From (2), (8) and (11) it is seen that in the SA the angle dependences of the TDF's of "like" and "unlike" triplets are the same, viz.

$$
\begin{aligned}
& h_{1}^{\prime}(r, r, \vartheta) \\
& =g_{1}(2 r \sin (\vartheta / 2)) / \int_{-1}^{1} g_{1}(2 r \sin (\vartheta / 2) \mathrm{d} \cos \vartheta,
\end{aligned}
$$

where the subscript 1 at $h_{1}^{\prime}$ refers to the likeness of the ions forming the pair in the shell.

In Figs. 2 and 3 the functions $h_{\mathrm{uu}}, h_{11}$ and $h_{1}^{\prime}$ for given values of $r$ are plotted vs. $\cos \vartheta$. From the figures and the relations

$$
\begin{aligned}
& g_{\mathrm{ss}}(r, r, \vartheta)=2 G_{\mathrm{ss}}(r, r) h_{\mathrm{ss}}(r, r, \vartheta) \\
& g_{\mathrm{ss}}^{\prime}(r, r, \vartheta)=2 G_{\mathrm{ss}}^{\prime}(r, r) h_{1}^{\prime}(r, r, \vartheta),
\end{aligned}
$$

with $\mathrm{s}=\mathrm{u}$ or 1 , the TDF's $g_{\mathrm{uu}}, g_{\mathrm{uu}}^{\prime}, g_{\mathrm{ll}}$ and $g_{\mathrm{ll}}^{\prime}$ are obtained.

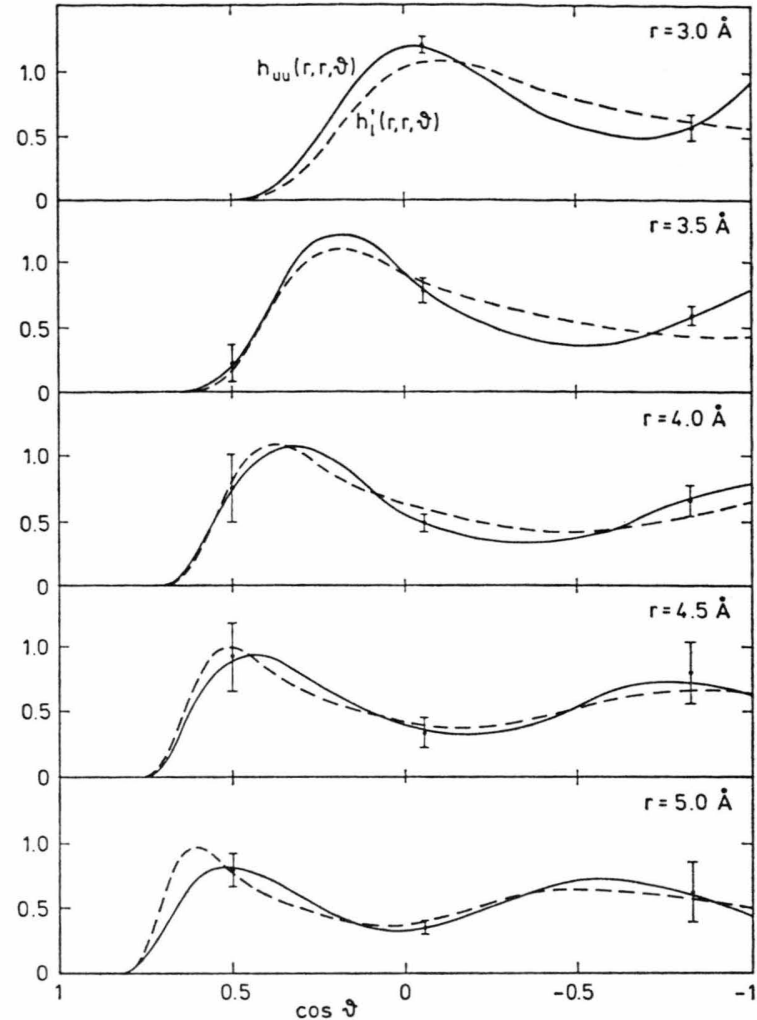

Fig. 2. Angle dependences of "unlike" TDF"s. The curves become horizontal lines, $1 / 2$ vs. $\cos \vartheta$, for noninteracting particles here and in Figure 3. Complete TDF's $g_{\text {uu }}(r, r, \vartheta)$ and $g_{\mathrm{uu}}(r, r, \vartheta)$ result by multiplication according to (13). For error bars see text.

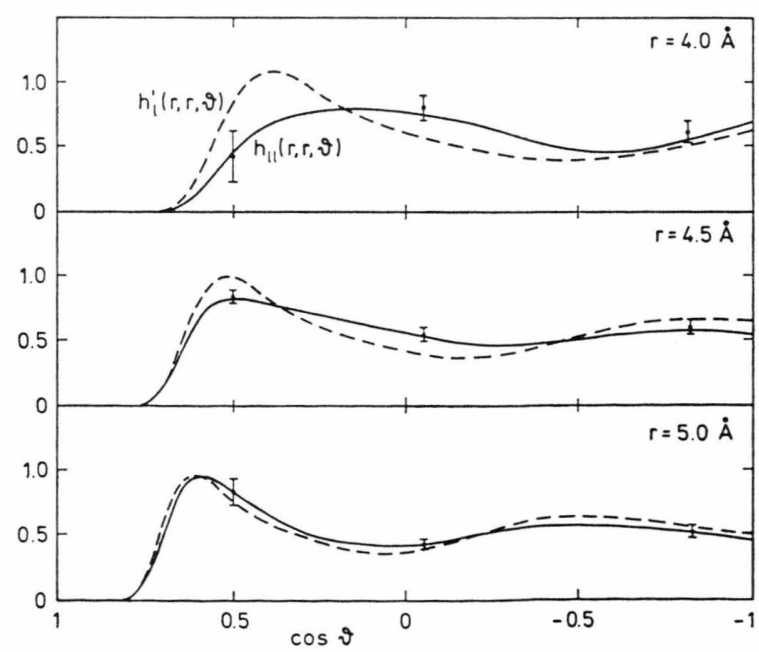

Fig. 3. Angle dependences of "like" TDF's. Complete TDF's $g_{11}(r, r, \vartheta)$ and $g_{11}^{\prime}(r, r, \vartheta)$ result by multiplication according to (13). For error bars see text. 
The knowledge of the PDF's was good enough (cf. [8]) to yield reliable curves $G_{\mathrm{sg}}^{\prime}(r, r)$ and $h_{1}^{\prime}(r, r, \vartheta)$. The curves $G_{\mathrm{ss}}(r, r)$ in Fig. 1 are also quite accurate, because the counting of pairs in shells $(r \pm 0.05 \AA)$ did yield numbers which are sufficiently large for good statistics. The curves $h_{\mathrm{ss}}(r, r, \vartheta)$, however, rely on the counting of pairs in the relatively narrow zones $(r \pm 0.05 \AA, \vartheta \pm$ $(\pi / 180))$, yielding less good statistics. In order to show the essential features, the statistical fluctuations have been smoothed out in the curves $h_{\mathrm{ss}}(r, r, \vartheta)$ shown in Figs. 2 and 3. The error bars mark the maximal deviations in the $\cos \vartheta$-ranges $0.5 \pm 0.05,-0.06 \pm 0.05$ and $-0.84 \pm 0.05$.

\section{Discussion of the Results}

A qualitative understanding of some features of the curves shown in Figs. 1, 2 and 3 may be conveyed as follows:

1. With increasing values of $r$ the functions $G_{\mathrm{uu}}(r, r)$ and $G_{\mathrm{uu}}^{\prime}(r, r)$ are becoming equal before becoming unity. The reason for this behaviour is the fact that the number of ions mediating between the three ions of a triplet is larger and rises more quickly with increasing distances than the number of ions mediating between two ions of a corresponding intrapair distance. Since the correlations are weaker the more ions are mediating, the triplet correlations vanish more quickly with increasing interparticle distances than the pair correlations. The functions $G_{11}(r, r)$ and $G_{11}^{\prime}(r, r)$ differ from zero only at relatively large values of $r$ and are therefore equal throughout.

[1] B. J. Alder, Phys. Rev. Lett. 12, 317 (1964).

[2] A. Rahman, Phys. Rev. Lett. 12, 575 (1964).

[3] S. Wang and J. A. Krumhansl, J. Chem. Phys. 56, 4287 (1972).

[4] H. J. Raveché, R. D. Mountain, and W. B. Street, J. Chem. Phys. 57, 4999 (1972).

[5] H. J. Raveché, R. D. Mountain, and W. B. Street, J. Chem. Phys. 61, 1970 (1974).
2. At small values of $r, G_{\mathrm{uu}}(r, r)$ is smaller than $G_{\mathrm{uu}}^{\prime}(r, r)$, i.e. the probability that three ions are very close to each other is in the average smaller than the product of the three probabilities of pairs with corresponding intrapair distances. The reason for this is that a small distance between two ions is in the average less probable if a third ion is by definition close to the two ions than if this requirement is not imposed.

3. With increasing values of $r$ the functions $h_{\mathrm{uu}}(r, r, \vartheta), h_{11}(r, r, \vartheta)$ and $h_{1}^{\prime}(r, r, \vartheta)$ are becoming similar before becoming $1 / 2$. The reason is the same as given sub 1 .

4. The curves $h_{\mathrm{uu}}(r, r, \vartheta)$ show more structure than the curves $h_{1}^{\prime}(r, r, \vartheta)$, and the curves $h_{11}(r, r, \vartheta)$ show less structure. While $h_{1}^{\prime}(r, r, \vartheta)$ depends only on the pair correlations between the ions in the shell, for $h_{\mathrm{ss}}(r, r, \vartheta)$ it is of relevancy that an ion is in the center of the shell. The Coulomb force of the central ion attracts the unlike ions in a shell and repels the like ions. This allows the unlike ions in the shell to settle at closer angular distances than would be expected in the SA, and viceversa for the like ions.

5. As was demonstrated in [8], the peaks at $\vartheta=$ $\pi / 2$ and $\vartheta=\pi$ of the $h_{\mathrm{uu}}(r, r, \vartheta)$-curve for $r=3 \AA$ correspond to the $\mathrm{f} \mathrm{c} \mathrm{c} \mathrm{structure} \mathrm{of} \mathrm{crystalline} \mathrm{KCl}$. From the PDF's alone, i.e. within the SA, the second peak at $\vartheta=\pi$ would not have been expected.

The paper demonstrates that the information about the structure of a molten salt remains limited if only the PDF's are known and becomes richer with the knowledge of the TDF's.

We wish to thank Prof. F. Forstmann for helpfull discussions during his stay at Mainz.

[6] M. Tanaka and Y. Fukui, Progr. Theor. Phys. 53, 1547 (1975).

[7] R. Block and W. Schommers, J. Phys. C, 8, 1997 (1975).

[8] L. Schäfer and A. Klemm, Z. Naturforsch. 34a, 993 (1979).

[9] A. D. J. Haymet, S. A. Rice, and W. G. Madden, J. Chem. Phys. 74, 3033 (1981). 